\title{
Author Correction: Ultrafast probes of electron-hole transitions between two atomic layers
}

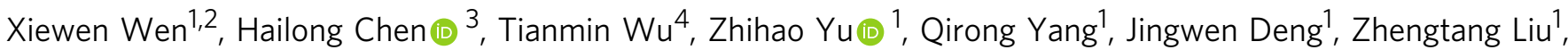
Xin Guo', Jianxin Guan ${ }^{1}$, Xiang Zhang ${ }^{2}$, Yongji Gong ${ }^{2}$, Jiangtan Yuan ${ }^{2}$, Zhuhua Zhang ${ }^{2}$, Chongyue $\mathrm{Yi}^{5}$, Xuefeng Guo (1) ${ }^{1}$, Pulickel M. Ajayan², Wei Zhuang', Zhirong Liu', Jun Lou² \& Junrong Zheng ${ }^{1}$

Correction to: Nature Communications https://doi.org/10.1038/s41467-018-04291-9, published online: 10 May 2018

The original version of this Article omitted an affiliation of Xiewen Wen: 'College of Chemistry and Molecular Engineering, Beijing National Laboratory for Molecular Sciences, Peking University, Beijing 100871, China'. This has been corrected in both the PDF and HTML versions of the Article.

Published online: 07 June 2018

(i) Open Access This article is licensed under a Creative Commons Attribution 4.0 International License, which permits use, sharing, adaptation, distribution and reproduction in any medium or format, as long as you give appropriate credit to the original author(s) and the source, provide a link to the Creative Commons license, and indicate if changes were made. The images or other third party material in this article are included in the article's Creative Commons license, unless indicated otherwise in a credit line to the material. If material is not included in the article's Creative Commons license and your intended use is not permitted by statutory regulation or exceeds the permitted use, you will need to obtain permission directly from the copyright holder. To view a copy of this license, visit http://creativecommons.org/licenses/by/4.0/.

(C) The Author(s) 2018

\footnotetext{
${ }^{1}$ College of Chemistry and Molecular Engineering, Beijing National Laboratory for Molecular Sciences, Peking University, Beijing 100871, China. ${ }^{2}$ Department of Materials Science and NanoEngineering, Rice University, 6100 Main Street, Houston, TX 77005-1892, USA. ${ }^{3}$ Beijing National Laboratory for Condensed Matter Physics, CAS Key Laboratory of Soft Matter Physics, Institute of Physics, Chinese Academy of Sciences, Beijing 100190, China. ${ }^{4}$ Department of Chemical Physics, University of Science and Technology of China, Hefei, Anhui 230026, China. ${ }^{5}$ Department of Chemistry, Rice University, 6100 Main Street, Houston, TX 77005-1892, USA. ${ }^{6}$ State Key Laboratory of Structural Chemistry, Fujian Institute of Research on the Structure of Matter, Chinese Academy of Sciences, Fuzhou, Fujian 350002, China. Correspondence and requests for materials should be addressed to

W.Z. (email: wzhuang@fjirsm.ac.cn) or to Z.L. (email: liuzhirong@pku.edu.cn) or to J.L. (email: jlou@rice.edu)

or to J.Z. (email: junrong@pku.edu.cn or (email: zhengjunrong@gmail.com)
} 